\title{
Poincaré maps define topography of Vlasov distribution functions consistent with stochastic dynamićs
}

\author{
A. D. Bailey III, a) P. M. Bellan, and R. A. Stern ${ }^{\text {b) }}$ \\ California Institute of Technology, Pasadena, California 91125
}

(Received 3 January 1995; accepted 14 April 1995)

\begin{abstract}
In a recent paper [A. D. Bailey et al., Phys. Rev. Lett. 34, 3124 (1993)], the authors presented direct planar laser induced fluorescence measurements of the oscillatory ion fluid velocity field in the presence of a large amplitude drift-Alfven wave. Surprisingly, the measured speeds were an order of magnitude lower than predicted by standard fluid theory, yet the flow pattern was consistent with the fluid theory. A new model, based on the connection between stochasticity and bulk behavior, is presented which gives insights into the cause of this behavior. It is shown that when particle motion is stochastic, invariant sets of a 'Poincare map' define a flat-topped particle distribution function consistent with both the electromagnetic field driving the Vlasov equation and the fine-scale single particle dynamics. The approach is described for the general case and explored for a slab model of the observed drift wave. (c) 1995 American Institute of Physics.
\end{abstract}

\section{INTRODUCTION}

The understanding of plasma physics would be very limited if a complete description of the plasma were needed to explain observed phenomena. By necessity a wide range of theoretical methods have been used to address the behavior of plasmas. Every approach is based on a set of approximations that simplify, obscure or ignore certain aspects of reality in order to shed light on the physics of interest. Very different theories may prove successful at explaining a number of observations from a plasma while a self-consistent description encompassing all the results remains elusive. This paper will focus on the connection between microscopic particle behavior and macroscopic plasma parameters described by two fundamental approaches: single particle orbits and the Vlasov equation.

The Vlasov description of plasma depends on the concept of the continuous distribution function $f(x, v, t)$ of the number of particles with three dimensional spatial position $\mathbf{x}$ and velocity $\mathbf{v}$ at time $t$. Velocity moments of the Vlasov equation describe macroscopic plasma phenomena. Although this seems to be a complete description, the Vlasov equation and its moment equations do not provide sufficient insight into the physics underlying certain phenomena. This is because the critical physics is contained in a more microscopic point of view, namely the particle trajectories governed by the Lorentz equations. Though $f(\mathbf{x}, \mathbf{v}, t)$ depends on these trajectories, knowledge of $f(\mathbf{x}, \mathbf{v}, t)$ does not prescribe these trajectories; for example, a Maxwellian distribution could equally describe unmagnetized particles with straight line orbits or magnetized particles with cyclotron orbits. Another example occurs when orbit stochasticity affects $f(\mathbf{x}, \mathbf{v}, t)$ resulting in particle 'heating'. In this situation, the distribution function provides an incomplete description because $f(\mathrm{x}, \mathrm{v}, t)$ cannot be 'inverted' to indicate that stochastic dy-

\footnotetext{
a) Present address: Plasma and Materials Technologies, Chatsworth, California 91311.

b) Permanent address: University of Colorado, Boulder, Colorado 80309.
}

namics are the key to understanding the broadening of $f(\mathbf{x}, \mathbf{v}, t)$.

A familiar connection between the Vlasov and Lorentz equations will be exploited to interpret Poincare maps in terms of particle distribution functions. These distributions are shown to be consistent with both the fields in the Vlasov equation and the resulting particle dynamics. The implications of this approach will be explored by considering a situation relevant to recent experiments ${ }^{1-3}$ in which ions were stochastically heated by a monochromatic electrostatic wave propagating perpendicular to a static magnetic field. The analysis of the stochastic particle dynamics for this heating in plasmas provides a framework for the new results. The primary result is that the stochastic nature of particle phase space trajectories results in a broadened and flattened particle distribution function in velocity space; this is analogous to the way stochastic mixing of field lines in magnetic braiding causes flattening and broadening of the magnetic flux profile in configuration space. ${ }^{4}$

\section{PLÄSMA HEATING DUE TO STOCHASTIC PARTICLE DYNAMICS}

It has long been known that the stochastic dynamics of single particles is related to 'heating' of plasmas. In order to analyze this mechanism, particle dynamics are typically investigated in simplified wave fields chosen to model a specific physical situation. Then the equations (sometimes a reduced set or a related mapping) are solved numerically and plotted graphically to provide a visual determination of parameters of interest and support hypotheses about plasma behavior. Zaslavskii ${ }^{5}$ and Rechester ${ }^{6}$ proposed the connection between heating in plasmas and the abrupt appearance of stochastic particle dynamics in the case of one traveling plane wave perturbed by another wave. Smith and Kaufman ${ }^{7,8}$ pointed out the existence of stochastic particle dynamics in a single electrostatic wave traveling obliquely to a magnetic field. They proposed using an ion acoustic wave satisfying a stochastic criterion to heat the tail of an ion distribution. Interest in heating ions in thermonuclear fusion 
devices by waves propagating perpendicular to a magnetic field, i.e., propagating towards the center of a tokamak, motivated further research in the dynamics of particles in a single wave propagating across a static magnetic field. Fukuyama ${ }^{9}$ studied the stochastic dynamics of ions in a plane monochromatic electrostatic wave perpendicular to a static magnetic field near an ion cyclotron harmonic. Karney ${ }^{10-12}$ studied the same situation in more detail for lower hybrid wave heating of fast ions at frequencies that were many times the cyclotron frequency. The same set of equations have often been studied analytically and numerically to describe particle heating in different wave amplitude and frequency parameter limits, ${ }^{3,13,14}$ in magnetic fields with shear ${ }^{15}$ and with relativistic effects. ${ }^{16}$ Stochastic ion heating has also been observed in plasma simulations of an inhomogeneous plasma. ${ }^{17}$

A connection between the stochastic dynamics of single ions in applied fields and the ion temperature is made heuristically in most studies. Convincing arguments based on intuition often appear in descriptions of how stochasticity causes heating. Similarly, knowledge gained from studying the stochastic dynamics is used to propose plausible heating models without making an explicil connection between the single particle dynamics and the broadened velocity distributions which must be the result of the proposed heating. These phrases are typical: particles 'random walk' from resonance to resonance, "(we) may then expect the particle to be able to move from the vicinity of one resonance to the vicinity of another," it "should be expected that diffusion of phase points from a cell to another cell takes place," "qualitative picture suggested by" calculations in which "an ion whose velocity satisfies (a condition) moves stochastically, ranging over that portion of (phase space)." these qualitative arguments with numerical calculations of the trajectories of a number of particles and then construct ion distribution functions which do indeed show ion heating (8 ions in Ref. 7, 10 in Ref. 9, 50 in Ref. 11, 100 in Ref. 8, 512 in Ref. 18, 10,000 in Ref. 19, 200,000 in Ref. 2).

Foreshadowing the results presented here, Smith and Kaufman ${ }^{8}$ used an alternative approach. They attempted to determine the equilibrium ion distribution by modifying an initially Maxwellian distribution function. The following argument based on experience with the single particle dynamics was used: "The modification (of the distribution) is accomplished by successively considering semicircular annuli in $v_{\perp}, v_{z}$ space, each of which represents particles with a small range of speeds. For each annulus the Maxwellian is integrated over the stochastic portion of the annulus to find the total number of stochastic ions in the annulus. This number is then redistributed over the stochastic portion of the annulus" (Ref. 8, italics added). The idea is that the ions will spread themselves uniformly within a stochastic region of phase space instantly whenever they enter it. Since there are more particles with low speeds initially and the stochastic regions extend from low to high speed regions, the phase space spreading quickly increases the number of particles with higher speeds, thereby heating the ions. Implicit in this model is the idea that the non-periodic stochastic particle motion caused by the wave does not disturb the periodic motion required to support the wave. Yet significant stochastic heating of the distribution function ${ }^{3,2,20,21}$ implies significant numbers of stochastic particles. The effect of these particles on the wave and related consistency problems have been too difficult to address theoretically in the past.

In contrast, experiments are naturally self-consistent. The primary focus of experimental work has been to establish the following three features of stochastic heating: (1) the temperatures are hotter with the wave than without the wave or hotter than expected from standard mechanisms, (2) the heating displays a threshold behavior corresponding to the onset of stochasticity in model equations, and (3) the heating rate is fast compared to non-stochastic processes. Doveil ${ }^{22}$ measured stochastic electron heating in a standing wave using an electrostatic analyzer. The unique capabilities of laser induced fiuorescence (LIF) have been used to study stochastic ion motion with unprecedented detail. Stochastic ion heating has been observed in a number of plasma waves: ion Bernstein waves in an Ar linear magnetized plasma, ${ }^{20}$ driftAlfvén waves in Ar tokamak discharges ${ }^{3}$ and electrostatic ion cyclotron waves in a $\mathrm{Ba} \mathrm{Q}$-machine plasma, ${ }^{21}$ all using LIF techniques. Consistency issues related to the synergy between the dielectric response of the plasma which supports a wave and the stochastic particle dynamics which heat the plasma have only recently begun to be measured. ${ }^{23,24}$

Experiments on the drift waves in Caltech's Encore tokamak highlight the importance of stochastic particle dynamics within a plasma that can still be described using a relatively simple model. The waves measured in Encore were identified using a cold-ion two-fluid model, ${ }^{25}$ yet the ion temperatures are observed to be anomalously hot due to stochastic ion motion in the coherent waves. ${ }^{3}$ Planar laser induced fluorescence (PLIF) plasma measurements ${ }^{1}$ showed that beyond the stochastic threshold for ion heating, the ion fluid fiow pattern in the coherent drift wave is well described by the fluid equations, but the magnitude of the velocities are an order of magnitude smaller than predicted by the fluid theory. The new theoretical approach described below may lead to a clearer understanding of how the complex ion dynamics and simple periodic behavior of the plasma are intertwined in a self-consistent way.

\section{SELF-CONSISTENT DISTRIBUTIONS FROM POINCARE MAPS}

As the discussion above demonstrates, the selfconsistent relationships between stochastic single particle dynamics and macroscopic (often periodic) plasma parameters are not fully understood. One solution to this problem is to find a distribution function consistent with the fields that cause stochastic particle motion. The velocity moments of such a distribution can then be used to find macroscopic plasma parameters. The idea is to begin with mean fields known to exist in a situation, study the dynamics of single particles in those felds, then derive a distribution function consistent with the fields using the particle dynamics, therefore ensuring consistency with single particle stochasticity. This approach can also address the question of whether stochastic dynamics are the cause (in a temporal sense) or the result of heating. We will show that this question is ill-posed 
since stochastic dynamics is the underlying reason why the distribution function is broadened ${ }^{26}$; there is no temporal cause and effect relationship since one cannot have stochastic dynamics without broadened distribution functions.

The simplifications in this approach are identical to those needed to derive the Vlasov equation;

$$
\frac{\partial f}{\partial t}+\mathbf{v} \cdot \frac{\partial f}{\partial \mathbf{x}}+\frac{q}{m}(\mathbf{E}+\mathbf{v} \times \mathbf{B}) \cdot \frac{\partial f}{\partial \mathbf{v}}=0 ;
$$

we emphasize that in order to derive the Vlasov equation, one must assume that $f$ is analytic (i.e., continuous and smooth). This assumption of analyticity is reasonable since $f$ is a physically measurable quantity. A standard method for obtaining approximate solutions to the Vlasov equation for prescribed fields is to use the method of characteristics in an approximate manner by integrating over unperturbed orbits. ${ }^{27-29}$ In this technique the perturbation to an initial distribution function due to a wave is found by integrating the perturbation along unperturbed orbits which are presumably known analytically. This method is not accurate when the perturbed trajectories differ substantially from the unperturbed orbits, e.g., when there are effects of trapped particles in nonlinear Landau damping ${ }^{30}$ or in situations where the perturbed orbits become stochastic, exponentially diverging from the unperturbed orbits.

An alternate approach-which is the main point of this paper--is to use directly the fact that the Vlasov equation is simply a statement that the distribution function is constant along its characteristics in phase space. These characteristics are just the particle orbits prescribed by integrating the Lorentz equation, i.e.,

$$
\begin{aligned}
& \mathbf{x}=\mathbf{x}(0)+\int_{0}^{t} \mathbf{v}\left(t^{\prime}\right) d t^{\prime}, \\
& \mathbf{v}=\mathbf{v}(0)+\frac{q}{m} \int_{0}^{t}\left[\mathbf{E}\left(t^{\prime}\right)+\mathbf{v}\left(t^{\prime}\right) \times \mathbf{B}\left(t^{\prime}\right)\right] d t^{\prime} .
\end{aligned}
$$

If the distribution function is known at $t=0$ and the characteristics given by Eq. (2) are known, the Vlasov equation is effectively solved because the value of the distribution function at any time $t$ is $f(\mathbf{x}, \mathbf{v}, t)=f(\mathbf{x}(0), \mathbf{v}(0), 0)$. A distribution function which satisfies the Vlasov equation can be constructed by assigning values to the distribution function over any surface in phase space normal to all the characteristics, thus fixing a value for each characteristic. This is a generalization of the familiar statement that any function of the constants of motion is a distribution function satisfying the Vlasov equation.

Thus, the errors inherent in integration over unperturbed orbits are avoided since the orbits are calculated directly. In a system with well-defined constants of motion, the characteristics typically form smooth surfaces which foliate phase space. In stochastic cases the characteristics typically do not form such surfaces, but the characteristics are still given by Eq. (2). The essential problem then is to develop a way to understand what distributions look like when the characteristics do not form foliating surfaces.

The standard way of viewing the dynamics of single particle orbits is the Poincare surface of section. Each time a particle trajectory passes through a chosen phase space plane (the 'surface of section') a point is plotted in the plane where the particle 'punctures' this plane. Since a particle trajectory maps out a characteristic of the Vlasov equation, all punctures on the plane by a single particle trajectory locate points in phase space with the same distribution function value. The points in the plane found by following the particle for infinite positive and negative time constitute a set of points in the plane which are invariant under a Poincaré map (a map of the plane to itself under the particle dynamics). Thus invariant sets of a Poincare map are level sets of the distribution function; i.e., the distribution function has the same value for the set of all puncture points made by a single particle. Thinking of the distribution function as an altitude function over the surface of section plane, Poincare surface of section plots provide unmarked 'topographic maps' of the distribution function consistent with the prescribed fields. The maps are unmarked because any choice of constants assigned to the respective characteristics will. satisfy the Vlasov equation.

This interpretation of the Poincare map gives new significance to a surface of section plot as a window onto the physical world and not only as a geometric display of the mathematical dynamics. If the axes of a Poincare section are chosen to be velocities and/or positions (rather than more abstract variables constructed purely for mathematical purposes), a Poincaré plot can be viewed with a distribution function in mind. The missing link to make this picture truly self-consistent is to determine the values of the distribution function in a given plane which not only satisfy Vlasov's equation, but also Maxwell's equations with the same fields. The Poincare map shows where the distribution values must be the same, but it does nothing to set those values. Further discussion on this point will be postponed until the end of the paper. First, the implications of this new viewpoint are explored for a particular case relying on the assumption that the self-consistent velocity distribution is similar to a Maxwellian; it has large values for small speeds and decreases monotonically to zero at large speeds.

\section{IMPLICATIONS FOR A MODEL OF THE DRIFT WAVE}

We assume a straight uniform magnetic field

$$
\mathbf{B}=B_{0} \hat{\mathbf{z}}
$$

and consider particle motion in a perpendicularly propagating monochromatic plane electrostatic wave with electric field

$$
\mathbf{E}(y, t)=\tilde{\phi} k \sin (k y-\omega t) \hat{\mathbf{y}} \text {. }
$$

This is a simple slab model of the drift-Alfvén wave observed in the Caltech Encore tokamak ${ }^{1-3,25}$ (the experimentally observed wave has poloidal mode number $m=2$, the slab model coordinate $y$ corresponds to the poloidal direction).

The Hamiltonian form of the Lorentz equation in the fields prescribed by Eqs. (3) and (4) can be expressed as

$\dot{q}=p$, 

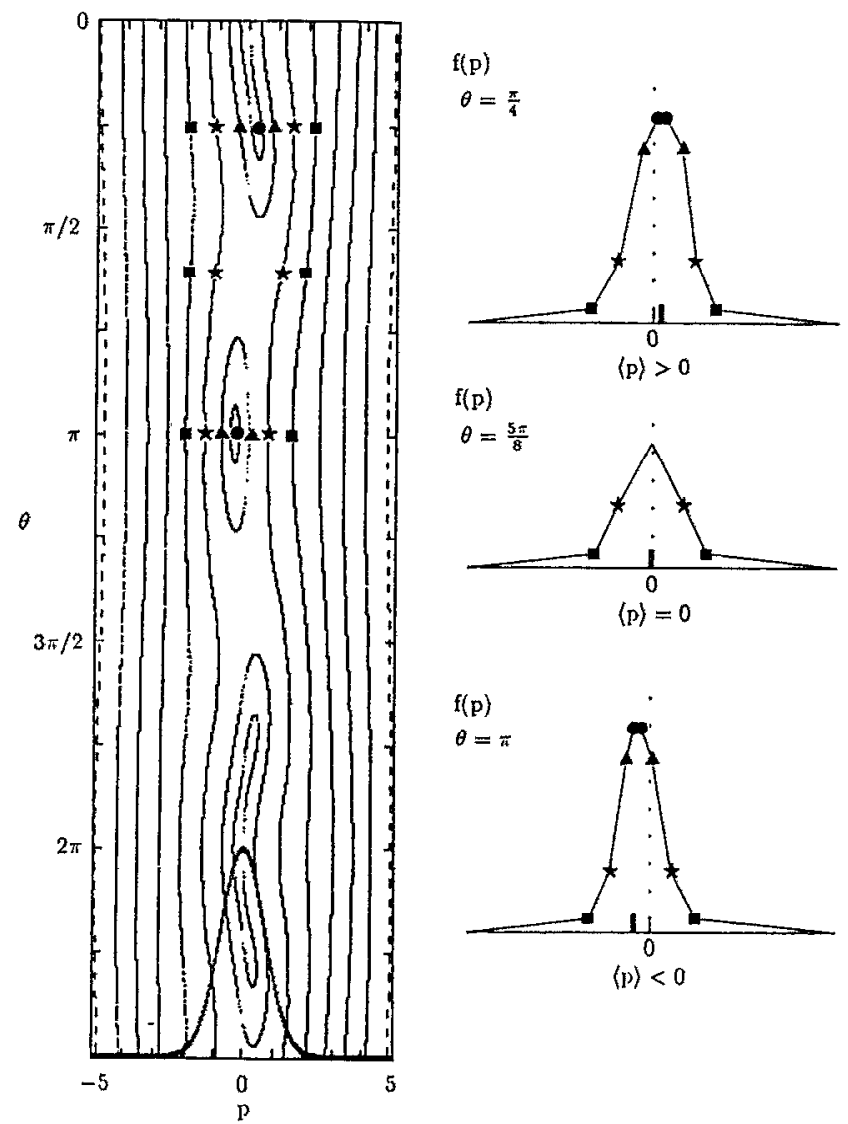

$\langle\mathrm{p}\rangle>0$

$\mathrm{f}(\mathrm{p})$

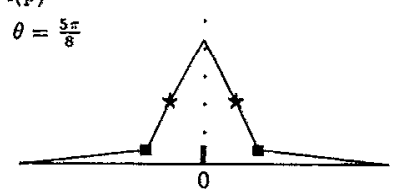

$\langle p\rangle=0$

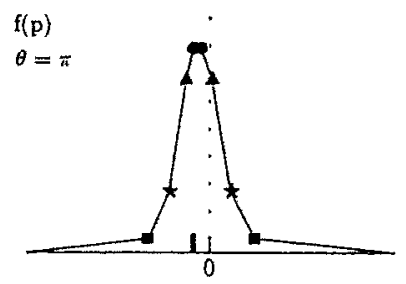

(p) $<0$

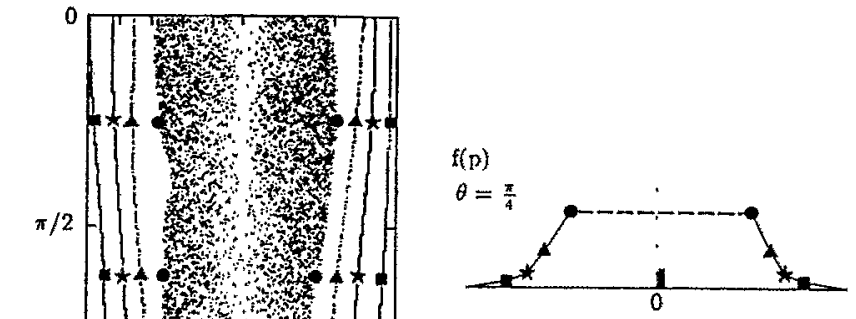

(p) $>0$

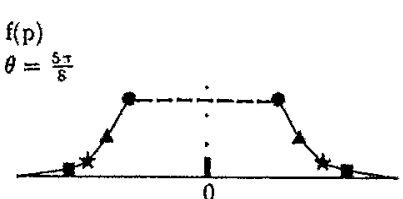

$\langle p\rangle=0$

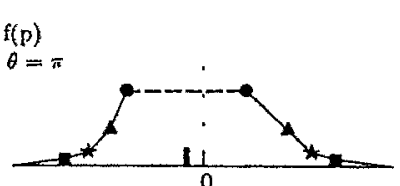

(p) $<0$

FIG. 1. Poincaré section in $q=0$ plane for $\alpha=0.4$ with $\nu=0.4567$. Each symbol marks a crossing of a single particle in the wave phases corresponding to the unnormalized distribution functions. A normalized Maxwellian corresponding to $1 \mathrm{eV}$ is plotted on the Poincare section for perspective.

$$
\dot{p}=-q+\alpha \sin (q-\nu t) .
$$

Here time and space have been normalized to $\omega_{c i}$ and $k$ respectively, the ratio of the characteristic frequencies is $\nu=\omega / \omega_{c i}$, the normalized wave amplitude is $\alpha=k^{2} \tilde{\phi} / \omega_{c i} B_{0}$ and in normalized units the velocities are $v_{x}=q$ and $v_{y}=p$. For parameters relevant to the drift waves in Encore $\nu \approx 0.5, \alpha \approx 1$.

Equations (5) have been thoroughly discussed for the high frequency situation ( $\nu \gg 1$ ): both the $\alpha \gg 1$ case and the $\alpha \ll 1$ have been analyzed, ${ }^{14,10-12,31,32}$ and also $\alpha \ll 1$ cyclotron harmonics ( $\nu=$ integer $>1$ ) have been analyzed ${ }^{9}$ as well as sub-harmonics ( $\nu=$ rational $>1$ ). ${ }^{17}$ Somewhat less attention has been given to the low frequency limit $(\nu<1)$; however this has been discussed for situations where $\nu \ll 1$ and $\alpha \ll 1$ or $\alpha \gg 1,{ }^{13}$ and aspccts of the nonlinear behavior in the $\nu<1, \alpha \gg 1$ limit have been studied qualitatively and numerically. ${ }^{3.33}$ As the normalized wave amplitude $\alpha$ increases to $\sim 0.7$ the dynamics become stochastic in a large region of phase space near $(q, p)=(0,0)$. Despite the physical significance of this situation, there has not been a clear treatment of the transition to chaos for $\nu \leqslant 1, \alpha \sim 1$ which includes the drift waves in Encore.

Poincaré sections for $\nu=0.4567$ are displayed in Figs. 1 and 2 . These figures display eight particle trajectories found
FIG. 2. Poincare section in $q=0$ plane for $\alpha=0.95$ with $\nu=0.4567$. Each symbol marks a crossing of a single particle in the wave phases corresponding to the unnormalized distribution functions. A normalized Maxwellian corresponding to $6 \mathrm{eV}$ is plotted on the Poincare section for perspective.

by directly integrating Eq. (5) using a fifth order Numerov scheme $^{34}$ for $\approx 1000$ wave periods. Before choosing the plane to display the Poincare section, the dimension of the phase space must be extended by a phase space variable which contains time. Two Poincare section plots in the $q=0$ plane in the $(q, p, \theta=v t)$ phase space are displayed in Figs. 1 and 2 for two values of $\alpha$. In these plots $\theta$ is the phase of the wave. From the viewpoint set out in the last section, the 'curves' in the Poincare section are level sets of a distribution function $f(q=0, p, \theta)$ or equivalently $f\left(v_{x}=0, v_{y}, \theta\right)$. When $\alpha>0$, but below the stochastic threshold, particle heating is not expected and the level sets are smooth foliating curves in the plane (see Fig. 1). For $\alpha$ above threshold when heating is expected, low velocity particles with stochastic trajectories map out invariant sets which intersect large areas in the phase plane (see Fig. 2). Although this fact alone does not directly imply heating, a firmer connection bctween stochasticity and heating can now be made without resorting to a plasma simulation or Monte-Carlo methods. This viewpoint also has implications for other macroscopic plasma parameters.

Plausible distributions derived by interpreting the Poincaré maps of the dynamics of single particles with a Maxwellian-like distribution are easily drawn for $\alpha=0.4$ 
(see Fig. 1). A $1 \mathrm{eV}$ Maxwellian was used to label the characteristics for $\theta=\pi$. This fixes the distribution in the rest of the phase space. The width of the distribution is broadened in various phases of the wave (see $\theta=5 \pi / 8$ in Fig. 2). Since this broadening is periodic it would not be considered 'heating' in the usual sense of the term.

It is less clear how to draw distributions in the case when $\alpha$ is above the stochastic threshold (see Fig. 2). Due to the complicated, intertwined characteristics in the stochastic region, the distribution function would become discontinuous if each characteristic were given a different value. Experimentally this implies that any finite bandwidth measurement in that region of phase space would result in a single averaged value (assuming the stochastic region was evenly covered by the different level sets). Since the distribution must be analytic [cf. discussion after Eq. (1)], the values assigned to these intertwined stochastic characteristics must be identical to ensure continuity. To maintain continuity from the non-stochastic regions, the value chosen must equal that of the 'last' integrable characteristic. Both arguments lead us to postulate a distribution function with a flat central region, essentially a broadened distribution. In Fig. 2 a normalized 6 $\mathrm{eV}$ Maxwellian distribution was used to set the values of the level sets at $\theta=5 \pi / 8$. Once again, this choice determines the distribution in the rest of the phase space. Since the stochastic region spans all phases of the wave, the distribution is broadened in all phases of the wave. This increased temperature is qualitatively different from the phase dependent broadening below the stochastic threshold although it is the result of deterministic non-'random' particle orbits.

Since it is the appearance of the stochasticity that leads to distribution functions with flat central regions in all phases of the wave, it is appropriate to say this heating is caused (in a logical rather than temporal sense) by stochasticity. From this viewpoint the heating does not depend on an irreversible transfer of energy from the wave to the particles via collisions or other irreversible phenomena. Rather, these arguments lead one to propose that in order to have a large amplitude monochromatic wave propagating perpendicular to a constant magnetic field it is necessary that the distributions be broad. It is a misconception that the ions could be 'cold' in large amplitude waves propagating perpendicular to $\mathbf{B}$.

At this point it is worth comparing our analysis to previous, more traditional approaches, for example, those used by Karney ${ }^{12}$ and by Menyuk et al. ${ }^{35}$ both of whom postulated that in the steady-state limit $t \rightarrow \infty$, the distribution function becomes flat and time-independent due to stochasticity. They argued that stochastic regions are associated with wave damping and particle heating. In contrast, we have shown here (cf. vertical dependence in Fig. 2) that the distribution function is time-dependent and does not go to a steady-state as $t \rightarrow \infty$; instead it has undulating flattened regions whose width corresponds to particle 'heating'. Furthermore, the wave is not temporally damped, but instead coexists indefinitely 'with the undulating flat regions. Energy is not 'transferred' from the wave to the particles; it is just that the distribution function must be broad when the particles move in this large amplitude field.

In order to make his argument, Karney invoked ergodic- ity, the statistical assumption that the average of an ensemble of systems is equivalent to the time average of a single system. In contrast, the system we describe here is not ergodic, as can be seen by consideration of Fig. 2 . If one considers an ensemble of $N$ such systems with different initial conditions for the particles, each of the systems will generate a Poincare map that will be macroscopically identical (i.e., have the same undulating flat regions) but will differ microscopically (the individual punctures will not be identical). Thus, the ensemble average of these $N$ systems will produce maps that look like Fig. 2. On the other hand, if one takes the time average of Fig. 2 (i.e., averages over the vertical coordinate), information about the undulations will be lost. Thus, the average of an ensemble is not the same as the time average of a single system and so the ergodic assumption is not appropriate here.

Menyuk et al. calculated the time-dependent wave damping of a weakly magnetized Langmuir wave, taking into account finite Larmor radius effects, assuming unperturbed orbits and distinct wave particle resonances. To address self-consistent effects between the wave fields and particle orbits Menyuk et al. simply assumed that if wave particle resonances overlap, the distribution function flattens so that particles spread in phase space. In contrast, our approach assumes a steady-state wave, does not invoke the assumption of unperturbed orbits, and demonstrates why the distribution functions must be flattened.

In Ref. 1 we reported that the experimentally measured fluid velocity of ions with stochastic orbits (caused by a large amplitude drift wave) was an order of magnitude lower than predicted by simple $E \times B$ drift theory and showed that conventional finite Larmor radius theory (CFLR) ${ }^{36}$ gave the correct order of magnitude reduction in the fluid velocity. CFLR theory assumes both linearization of the Vlasov equation and integration along unperturbed orbits. Although these approximations are, in fact, not applicable when the orbits are stochastic, CFLR could still give a reasonable estimate of the reduction of the fluid velocity because the averaging depends primarily on the large spatial extent of the orbits which is also apparent in the stochastic case. Although CFLR and the Poincare maps provide different ways to understand the surprisingly low fluid velocity, neither approach explains the measurements quantitatively. The non-integrable regions visible in Fig. 2 are the stochastic velocity space analog to the CFLR spatial phase averaging. To further complicate matters, the experimental results are not directly comparable with the Poincare maps due to the inherent integration over two velocity components performed by the PLIF measurement technique and the limitations of using a three instead of seven-dimensional phase space where the ignored dimensions may be important in the experiment.

Some insight into the full distribution can be gained by looking at Poincare maps in the extended phase space $(q, p, \psi)$ where $\psi=q-\nu t$ is the wave phase. In this case the level sets of the distribution $f(q, p)$ corresponding to $f\left(v_{x}, v_{y}\right)$ may be displayed in any phase of the wave by constructing the Poincare plot in the $\psi=\psi_{o}$ plane. Figs. 3 and 4 show series of plots in eight phases of the wave for the same particles and values of $\alpha$ and $\nu$ used in Figs. 1 and 2 . 

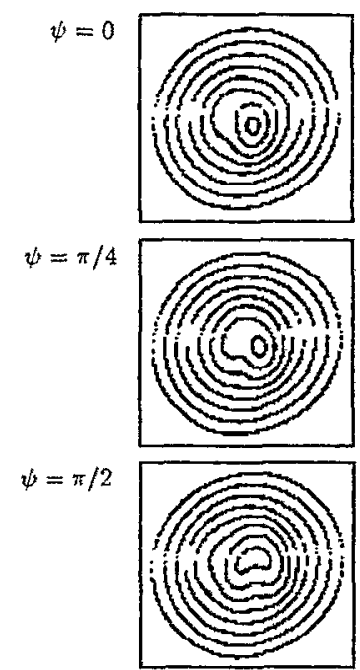

$\psi=3 \pi / 4$

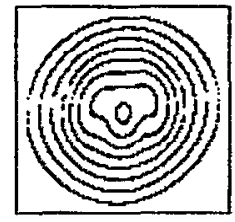

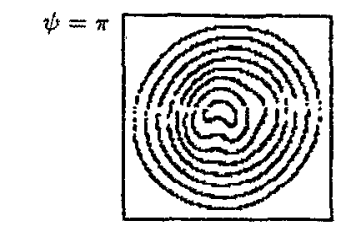

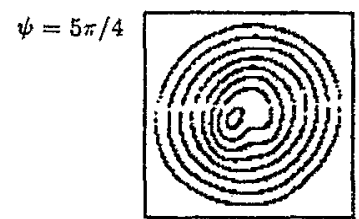

$\psi=3 \pi / 2$

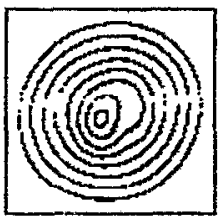

$\psi=7 \pi / 4$

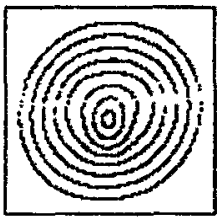

FIG. 3. Poincaré sections in constant $\psi$ planes for $\alpha=0.4$ with $\nu=0.4567$. Each box is 5 units square centered on the $(q, p)$ origin.

Thinking in terms of unmarked topographic maps, as $\alpha$ increases, the 'summit' of the distribution changes from a single oscillating peak for very low $\alpha$ (nearly concentric circles not pictured) to a complicated set of 'rolling hills' for intermediate $\alpha$ (Fig. 3) to a "stochastic mesa" for $\alpha$ near one (Fig. 4). Since the exact distribution value for each level surface is not known and the fluid velocity requires the first velocity moment of the distribution, it is difficult to predict the fluid velocity at each phase.

When valid, the fluid drift model ( $E \times B$ drifts, polarization drifts) should describe the motion of the 'center of mass' of these maps. The fluid drifts may be retrieved from Eq. (5) by taking a time derivative and then eliminating the first derivatives to obtain two coupled second order equations

$$
\begin{aligned}
& \ddot{p}+p=\alpha \frac{d \sin \psi}{d t}, \\
& \ddot{q}+q=\alpha \sin \psi .
\end{aligned}
$$

The drift approximation is equivalent to ignoring the $\ddot{q}$ and $\ddot{p}$ terms so that the normalized $E \times B$ drift corresponds to

$$
q(\psi)=\alpha \sin \psi
$$

while explicit evaluation of $d(\sin \psi) / d t$ shows that the polarization drift corresponds to

$$
p(\psi)=-\frac{\nu \alpha \cos \psi}{1-\alpha \cos \psi} .
$$

For small $\alpha$, these two drifts describe an elliptical motion of the 'center of mass' in the $q, p$ plane. This elliptical motion of the center of mass is visible in Fig. 3 (orbits not stochastic), but not obvious in Fig. 4 (orbits are stochastic). For
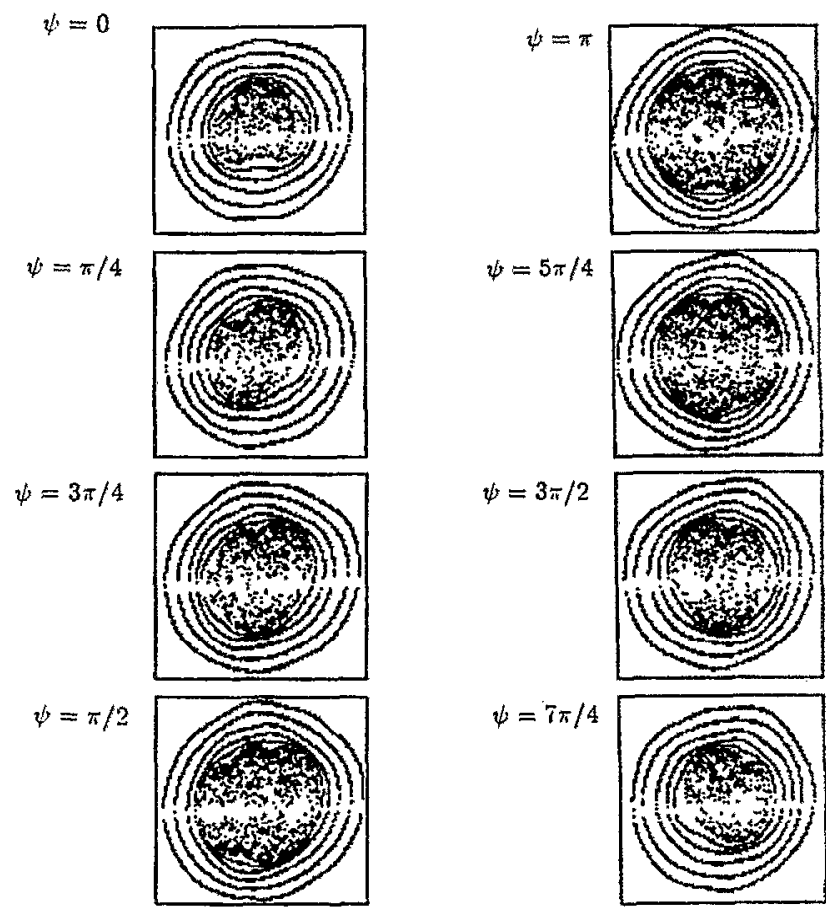

FIG. 4. Poincaré sections in constant $\psi$ planes for $\alpha=0.95$ with $\nu=0.4567$. Each box is 5 units square centered on the $(q, p)$ origin.

$\alpha=0.95$ the drift model would predict a maximum $q(\psi) \sim 0.95$ and a very large maximum $p(\psi) \sim \nu \alpha /(1-\alpha)$. Inspection of Fig. 4 shows that the magnitude of the elliptical motion of the 'center of mass' associated with the stochastic 'mesa' is, in fact, not large. Thus, the actual motion is significantly smaller than the prediction of naive drift theory (the problem, of course, is that for large $\alpha$, it is not selfconsistent to neglect $\ddot{q}$ and $\ddot{p}$ ). It is difficult to make an analytic estimate of this reduction (other than appealing to CFLR as discussed above) because of the complicated nature of the stochastic orbits.

\section{CONCLUSION}

An explicit connection between Poincaré maps of single particle dynamics and the continuous particle distribution function consistent with prescribed fields has been used to develop a new approach to the self-consistent waveparticle interaction. The method was shown by example to be especially promising when stochastic particle dynamics coexist with periodic fluid motion. The method is constructive, but requires few calculations compared to Monte-Carlo simulations and may lead quickly to new physical insight. Further work is required to provide a mechanism to determine if distributions with level sets constructed using a Poincaré section also satisfy Maxwell's equations (without simply stating that the electrons are Boltzmann) and thus support the prescribed wave fields. An iterative approach starting with the perturbed distribution calculated using integration over unperturbed orbits may provide a self-consistent solu- 
tion to the essential question: What distribution function is simultaneously consistent with a periodic wave field and stochastic parlicle motion?

\section{ACKNOWLEDGMENT}

This work was supported by the National Science Foundation under Grant No. PHY-9114146.

${ }^{1}$ A. D. Bailcy III, R. A. Stern, and P. M. Bellan, Phys. Rev. Lett. 34, 3124 (1993)

${ }^{2}$ J. M. McChesney, P. M. Bellan, and R. A. Stern, Phys. Fluids B 3, 3363 (1991).

${ }^{3}$ J. M. McChesney, R. A. Stern, and P. M. Bellan, Phys. Rev. Lett. 59, 1436 (1987).

${ }^{4}$ T. H. Stix, Phys. Rev. Lett. 38, 833 (1973); A. B. Rechester and T. H. Stix, ibid. 36, 587 (1976).

${ }^{5}$ G. M. Zaslavskii and N. N. Filonenko, Zh. Éksp. Teor. 54, 1590 (1968); Sov. Phys. JETP 25, 851 (1968).

${ }^{6}$ A. B. Rechester and T. H. Stix, Phys. Rev. A.19, 1656 (1979).

${ }^{7}$ G. R. Smith and A. N. Kaufman, Phys. Rev. Lett. 34, 1613 (1975).

${ }^{8}$ G. R. Smith and A. N. Kaufman, Phys. Fluids 21, 2230 (1978).

${ }^{9}$ A. Fukuyama, H. Momota, R. Itatani, and T. Takizuka, Phys. Rev. Lett. 38, 701 (1977).

${ }^{10}$ C. F. F. Kàrney and A. Bers, Phys. Rev. Lett. 39, 550 (1977).

${ }^{11}$ C. F. F. Karney, Phys. Fluids 21, 1584 (1978).

${ }^{12}$ C. F. F. Karney, Phys. Fluids 22, 2188 (1979).

${ }^{13}$ J. F. Drake and T. T. Lee, Phys. Fluids 24, 1115 (1981).

${ }^{14}$ M. A. Malkov and G. M. Zaslavsky, Phys. Lett. A 106, 257 (1984).

${ }^{15}$ Y. Gell and R. Nakach, Phys. Fluids 23, 1646 (1980).

${ }^{16}$ H. Karimabadi, K. Akimoto, N. Omidi, and C. R. Menyuk, Phys. Fluids B 2, 606 (1990).

${ }^{17}$ H. Abe, H. Okada, R. Itatani, M. Ono, and H. Okuda, Phys. Rev. Lett. 53, 1153 (1984)

${ }^{18} \mathrm{~J}$.-Y. Hsu, K. Matsuda, M. S. Chu, and T. H. Jensen, Phys. Rev. Lett. 43. 203 (1979).
${ }^{19}$ H. Abe, H. Momota, R. Itatani, and A. Fukuyama, Phys. Fluids 23, 2417 (1980).

${ }^{20}$ F. Skiff, F. Anderegg, and M. Q. Tran, Phys. Rev. Lett. 58, 1430 (1987).

${ }^{21}$ F. Skiff, F. Anderegg, T. N. Good, P. J. Paris, M. Q. Tran, N. Rynn, and R. A. Stern, Phys. Rev. Lett. 61, 2034 (1988).

${ }^{22}$ F. Doveil, Phys. Rev. Lett. 46, 532 (1981).

${ }^{23}$ F. Skiff, F. Anderegg, T. N. Good, P. J. Paris, M. Q. Tran, N. Rynn, and R. A. Stern, Plasma Phys. Controlled Nucl. Fusion 31, 1569 (1989).

${ }^{24}$ A. Fasoli, F. Skiff, R. Kleiber, M. Q. Tran, and P. J. Paris, Phys. Rev. Lett. 70, 303 (1993).

${ }^{25}$ E. D. Fredrickson and P. M. Bellan, Phys. Fluids 28, 1866 (1985).

${ }^{26}$ Classical Hamiltonian chaos is reversible and therefore is not a true entropy-increasing 'heating' mechanism. Yet in many situations Hamiltonian chaus is indistinguishable from true random heating processes. Detailed LIF tagging measurements can reveal the existence of conserved quantities $^{21}$ which could be used to distinguish random from Hamiltonian motion.

${ }^{27}$ J. E. Drummond, Phys. Rev. 110, 293 (1958).

${ }^{28} \mathrm{R}$. Z. Sagdeev and V. D. Shafranov, Proceedings of the 2nd UN International Conference on Peaceful Uses Atomic Energy, Geneva (International Atomic Energy Agency, Vienna, 1958), Vol. 31, p. 118.

${ }^{29} \mathrm{M}$. N. Rosenbluth and R. Rostoker, Proceedings of the 2nd UN International Conference on Peaceful Uses Atomic Energy, Geneva (International Atomic Energy Agency, Vienna, 1958), Vol. 31, p. 144.

${ }^{30}$ T. O'Neil, Phys. Fluids 8, 2255 (1965).

${ }^{31}$ J.-Y. Hsu, Phys. Fluids 25, 159 (1981).

${ }^{32}$ Y. Terashima, J. Phys. Soc. Jpn. 60, 2210 (1991).

${ }^{33}$ D. W. Forslund, R. L. Morse and C. W. Nielson, Phys. Rev. Lett. 27, 1424 (1971); D. W. Forslund, R. Morse, C. Nielson, and J. Fu, Phys. Fluids 15, 1303 (1972).

${ }^{34}$ S. E. Koonin, Computational Physics (Benjamin/Cummings, Menlo Park, 1986), pp. 50 and 51.

${ }^{35}$ C. R. Menyuk, J. M. Dawson, V. K. Decyk, B. D. Fried, and G. J. Morales, Phys. Rev. Lett. 48, 1104 (1982).

${ }^{36}$ M. N. Rosenbluth, N. A. Krall, and N. Rostoker, Nucl. Fusion Suppl. Part 1, 143 (1962). 